\title{
GROWTH AND CHARACTERIZATION OF GARNET THIN FILMS FOR MAGNETOOPTICAL READOUT
}

\author{
J. M. DESVIGNES, F. CHEVRIER, J. Ben YOUSSEF, H. Le GALL, J. C. LEHUREAU*, \\ and J. P. CASTERA*
}

C.N.R.S.-Laboratoire Magnétisme-Optique des Solides-92195 MEUDON, France

*Thomson-CSF, LCR-91401 ORSAY, France

\section{ABSTRACT}

Magnetic tape offers potential capacities of $20 \mathrm{Mbits} / \mathrm{cm}^{2}$, while conventional readout heads are limited, particularly by the impossibility of integrating a large number of heads in a stack. A magneto-optical high resolution transducer, using thin garnet films, allows the exploration of the whole track width. These films, grown by liquid phase epitaxy, have to satisfy to some properties as : magnetization about 300 Gauss, high Faraday rotation and low uniaxial anisotropy (Ku). Films of YIG $B i_{x} G a_{y}$, YIG $B i_{x}(G a, A l)_{y}$ were grown from different melts composition.

As grown films present high Faraday rotation but also high uniaxial anisotropy. Annealings at several temperatures are necessary to decrease $\mathrm{Ku}$.

For gadolinium garnet films doped with $\mathrm{Bi}$ and $\mathrm{Pr}$, (Gd IG : Bi $\mathrm{Pr} \mathrm{Al}$ ), the required Faraday rotation and uniaxial anisotropy are obtained as grown, the presence of praseodymium decreasing the $\mathrm{Ku}$.

\section{INTRODUCTION}

Magnetic tape has a potential storage density (20 Mbits $/ \mathrm{cm}^{2}$ ) quite comparable to that of optical media. Furthermore it takes advantage of a long history of industrial development leading to low price high quality and availability. Tests on commercial VTR showed that the noise limitation to very high density lies in the head and playback amplifier noise rather than in the tape itself. Another strong limitation to high density recording on tape is the inability to integrate headstacks to a very high level. In order to record the full width of the tape in a single pass with a limited number of heads one has to scan the tape with a rotating drum. The most obvious method of scanning is transverse and was actually used in the 2 inches VTR. However the need to stabilize the tape when the head enters the track restricts the use of this scanning device to very wide tape. The most common method of scanning is oblique as are all modern VTR. The major drawback of helical scanning is the Ioss of azimuth when the tape dimension changes due to variation in humidity and temperature. Longitudinal readout offers many advantages but the number of tracks needed for a high capacity recorder ranges from 1000 to 2000 with 5 microns pitch. Whereas the most advanced project integrates 20 magnetic heads with 90 microns pitch for Static Digital Audio Tape (SDAT).

Optical readout offers a high signal to noise ratio especially at low head to tape speeds : magnetic signal power ranges between pico watts and nano Watts, whereas optical source power ranges between micro watts and milli Watts. The other advantage of optical readout is the possibility to image thousands of tracks on readily available integrated detectors. 5000 cells CCD are today available at a reasonable cost. The low longitudinal resolution of optic compared to magnetic heads is not a major drawback since the tape signal to noise ratio varies as the inverse of the longitudinal density squared and only the first power of track density. In other words, for the same area density a square bit form offers better depth magnetization of the tape and less spacing loos than a conventional elongated bit form.

A good compromise between track density and bit density is a typically 5 by 1 square microns bit cell. A dedicated optical system allows such resolution over the full width of a half inch tape.

A magneto-optical high resolution transducer [1,2] allows the exploration of the whole. track width if its characteristics satisfy following specifications : magnetization : $4 \pi \mathrm{M}=300$ Gauss (Experiments showed that the saturation magnetization should not exceed half the coercivity of the tape); Faraday rotation : $\Phi_{F}>10000 \% \mathrm{~cm}(\lambda=6328 \mathrm{~A})$; zero-field strip domain period Po $=3 \mu \mathrm{m}, 3$ which implies optimal characteristic length and uniaxial anisotropy $\left(K_{u}\right)$ of 0.13 and $3600 \mathrm{erg} / \mathrm{cm}^{3}$, respectively. The obtention of a high $\phi_{F}$ requires the use of Bismuth which induces a high $K$ due to the non-uniform distribution of this ion in the garnet dodecahedral site. This problem can be solved in two ways : either by proceeding to annealings which reduce $\mathrm{K}_{u}$ through migration of $\mathrm{Bi}$ ions, or by doping the films with ions that make magnetization planar.

\section{EXPERIMENT}

Epitaxy furnace is a three zones furnace controlled by computer. Substituted Yttrium and Gadolinium iron garnet layers were grown by LPE on one inch-diameter (III)。 - oriented GGG or substituted GGG substrates with a lattice constant of 12,383 and $12,498 \AA$ respectively. The substrate has a small tilting during the whole process to decrease the melt residual after dipping. At the end of growth process the substrate was rapidly removed from the melt and rotated with 900 rpm to spin off the melt. 
2-1. YIG: $\mathrm{Bi}_{\mathrm{x}} \mathrm{Ga}_{\mathrm{y}}(\mathrm{x}=0.26-0.32 ; \mathrm{y}=1.06-1.01)$

The films grown from these compositions have not large Faraday rotation $\left(\phi_{\mathrm{F}} \simeq 2000^{\circ} / \mathrm{cm}\right)$, but due to their high crystallographic quality they have been used to analyze the behaviour of the physical parameters after heat treatment. A set of as-grown films have been obtained at $1133^{\circ} \mathrm{K}$ and from Fig. 1, it is observed that whatever their thickness, the stripe period condition $P=3$ microns is not obtained. On the other hand, after quenching at different temperatures $(T=\$ 305-$ $1397-1473^{\circ} \mathrm{K}$ ) the value of $\mathrm{P}$ can be decreased. An increase of the saturation magnetizalion $4 \pi \mathrm{M}$ (from 243 to 350 Gauss) is associated with the gallium migration from tetrahedral to octohedral sites during the heat treatment [3]. Such an increase explains a part of the shift of $P$ with $T$ as observed in Fig. 1. The other part is due to the decrease of the uniaxial anisotropy when $T_{q}$ increases since $P$ is an increasing function of the characteristic length $I=(A K u)^{\frac{1}{2}} / \pi M^{2}$. That is the reason why in table 1 two samples of same $4 \pi \mathrm{M}$ (319 and 324 Gauss) but different $\mathrm{Ku}$ ( 4200 and. $12660 \mathrm{erg} . \mathrm{cm}^{-3}$ ) present strong difference for their characteristic length and therefore for $P_{0}$.

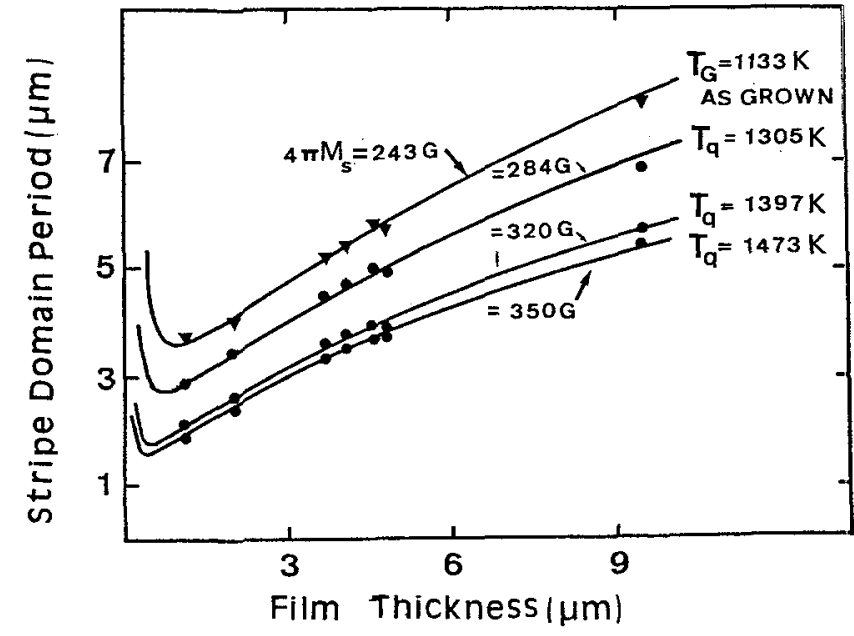

Fig. 1. Stripe Domain period dependence (Po) with film thickness ( $h$ ) for Grown and quenched films. Solid lines are calculated from "Shaw table".

\begin{tabular}{|c|c|c|c|c|c|}
\hline \multirow{3}{*}{$\begin{array}{l}\left.\mathrm{T}_{\mathrm{G}} \text { and } \mathrm{T}_{\mathrm{q}}{ }^{(\mathrm{q}} \mathrm{k}\right) \\
\mathrm{Ku}\left(\mathrm{erg} / \mathrm{cm}^{3}\right)\end{array}$} & \multirow{2}{*}{$\frac{\text { As grown } T_{G}}{1133}$} & \multicolumn{3}{|c|}{ Quenched at $T_{q}$} & As grown $T_{G}$ \\
\hline & & 1305 & 1397 & 1473 & 1150 \\
\hline & 8500 & 7000 & 42000 & 2000 & 12660 \\
\hline $4 \mathrm{~mm}_{\mathrm{s}}$ (Gauss) & 243 & 283 & 319 & 350 & 324 \\
\hline 1 (un) & 0,238 & 0,179 & 0,115 & 0,103 & 0,156 \\
\hline Q & 3,6 & 2,3 & 1,05 & 0,50 & 3,03 \\
\hline 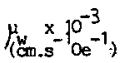 & 65 & 80 & 95 & 130 & 50 \\
\hline
\end{tabular}

Table 1 - Magnetic properties of YIG : Bi Ga films before and after heat treatment. Uniaxial anisotropy (Ku). Magnetization ( $4 \pi M s)$. Characteristic lenght (1). Quality factor $(Q)$ Domain mobility $\left(\mu_{w}\right)$.

2-2. $\mathrm{YIG}: \mathrm{Bi}{ }_{\mathrm{x}}(\mathrm{AlGa})_{\mathrm{y}}(\mathrm{x}=0.4-0.6 ; \mathrm{y}=1.02-1.1)$

Fig. 2 shows that whatever the growth temperature $\left(T_{G}\right)$ may be, no film prepared from this composition verifies the condition : Po $=3 \mu \mathrm{m}(4 \pi \mathrm{M}=217$ to 350 G). The caracteristic length $l$ is too large due to the high $K_{u}$. To avoid this, quenching at various temperatures $T$ has been performed. Only the results obtained with films of the series $\mathrm{Nr} .1581$ $(4 \pi M=217 \mathrm{G})$ at $\mathrm{T}=1471^{\circ} \mathrm{K}$ are plottêd in Fig. 2.9 It is well known that the uniaxial anisotropy $K$ is defined from two contributions : $K$ which is growth induced and $k S$ which is stressinduced anisotropy. It is observed from Fig. 3 that high $K$ values are mainly associated with growth-induced anisotropy. Annealing in an oxygen atmosphere can reduce significantly $K^{G}$ without change on $K^{S}$ (Fig.4). Some films exhibit a planar magnetization $\left(\mathrm{K}_{\mathrm{u}} \simeq 0\right)$ after quenching at too high temperature $\left(T=1473^{\circ} \mathrm{K}\right)$. Quenching at a lower temperature gives a very precise control of $K$ and therefore an approach of the selected characteristic length $(1=0.13 \mu)$. However, one of the specifications is not met. The Faraday rotation cannot exceed $4000 \% \mathrm{~cm}$ and, in spite of many attempts an increase of the magnetooptical effect is associated with a bad surface morphology of the films. When the bismuth oxide content is too large in the melt, the viscosity 
becomes too high and the quality of the film surface decreases. However, in the optical devices the defect concentration must be lower than 1 defect $/ \mathrm{cm}^{2}$, which cannot be reached with such solutions.
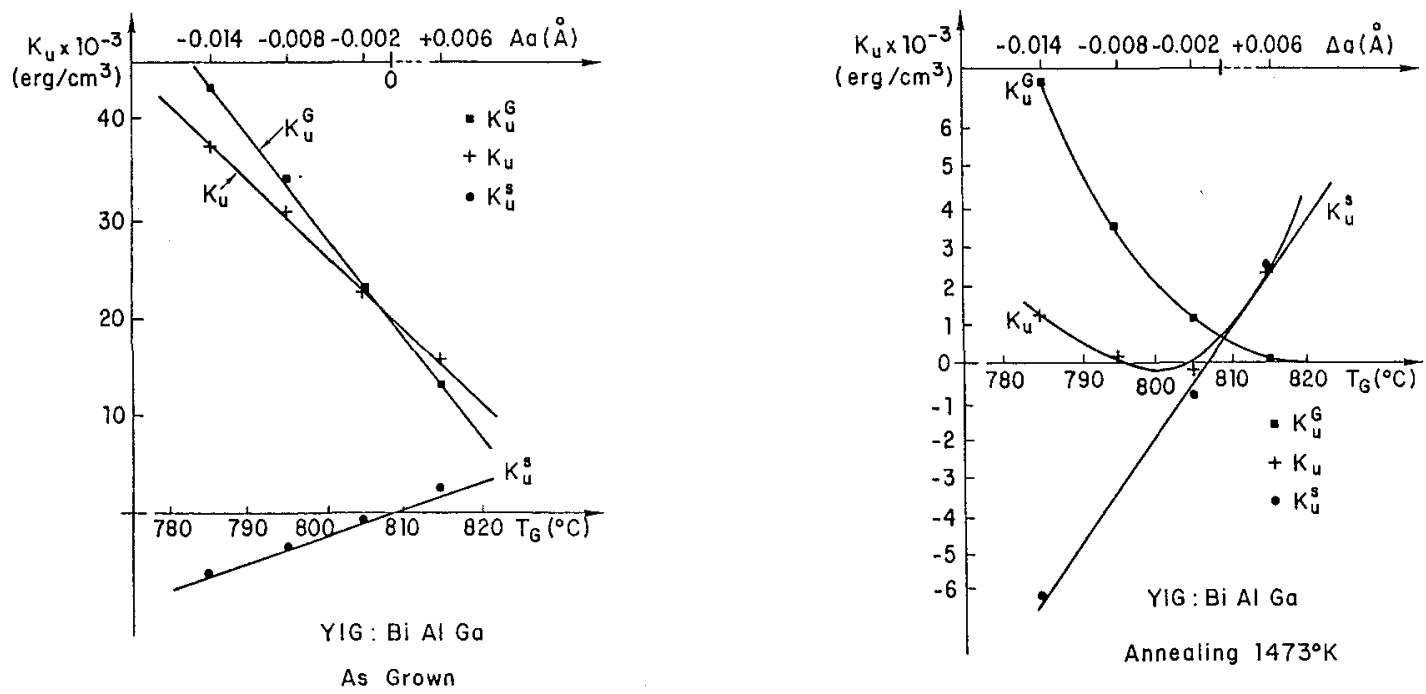

Fig. 3a 3b. Variation in $\mathrm{K}_{\mathrm{u}}$ as a function of growth temperature $\mathrm{T}_{\mathrm{g}}$ before and after quenching. $K_{u}^{S}=$ stress contribution, $K_{u}^{G}=$ growth contribution.

\section{2-3. Gd IG : Bi Pr Al}

Substituted - gadolinium garnet has been selected since higher Bi contents can be introduced in such a host [4]. Praseodymium ions has a high contribution to the Faraday rotation but its main effect is to reduce $K_{u}$ [5]. Similar result can be obtained with Nd ${ }^{3+}$ ions. The saturation magnetization is reduced to the selected value from the substitution of Fe by non magnetio ions. The best choice is obtained with $\mathrm{Al}^{3+}$ ions with a smaller content compared to $\mathrm{Ga}^{3+}$ in order to match the lattice parameters of the films and substrates.

\begin{tabular}{|c|c|c|c|c|c|c|c|c|c|c|c|}
\hline \multirow[b]{2}{*}{ MELT } & \multicolumn{4}{|c|}{ Melt ratio } & \multicolumn{6}{|c|}{ Film Composition } & \multirow[b]{2}{*}{$\begin{array}{l}\mathrm{T} \text { comp. } \\
(\mathrm{O})\end{array}$} \\
\hline & $\frac{\mathrm{Fe}+\mathrm{Al}}{\mathrm{Gd}+\mathrm{Pr}}$ & $\frac{\mathrm{Fe}}{\mathrm{Al}}$ & $\frac{\mathrm{Pb}}{\mathrm{Bi}+\mathrm{B}}$ & $\frac{\text { EGarnet }}{\text { Total }}$ & $\stackrel{T_{G}}{(0 k)}$ & Gd & $\operatorname{Pr}$ & $\mathrm{Bi}$ & $\mathrm{Fe}$ & Al & \\
\hline A & 20 & 8 & 1.5 & 0.10 & 1038 & 1.70 & 0.34 & 0.96 & 4.34 & 0.66 & 200 \\
\hline B & 18.1 & 8 & 1.5 & 0.11 & 1023 & 1.50 & 0.46 & 1.04 & 4.32 & 0.68 & 145 \\
\hline
\end{tabular}

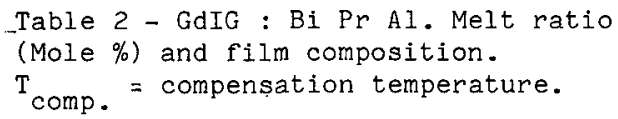

Two melt compositions have been investigated as reported in Table 2 as a function of mole \% ratios. The precise film compositions are obtained from electron microprobe analysis and the magnetic compensation temperature $T$ is deduced from Faraday rotation measurements at $6328 \mathrm{~A}$ wavelength. As shown in Fig. 4, the uniaxial anisotropy presents smaller values compared to the previous compositions due to $\mathrm{Pr}^{3+}$ ions which contribute to an in-plane magnetization. Substituted GdIG garnets have high Faraday rotation $\left(\phi_{F}=14000^{\circ} / \mathrm{cm}\right.$ at $\left.\lambda=6328 \mathrm{~A}\right)$ and since heat treatment is not necessary to adjust the magnetic parameters, these compounds are very interesting from manufacturing process point of view. The uniaxial anisotropy can be controled either from the growth temperature $\left(\mathrm{K}_{\mathrm{u}}\right.$ decreases with $\left.\mathrm{T}_{\mathrm{G}}\right)$ or

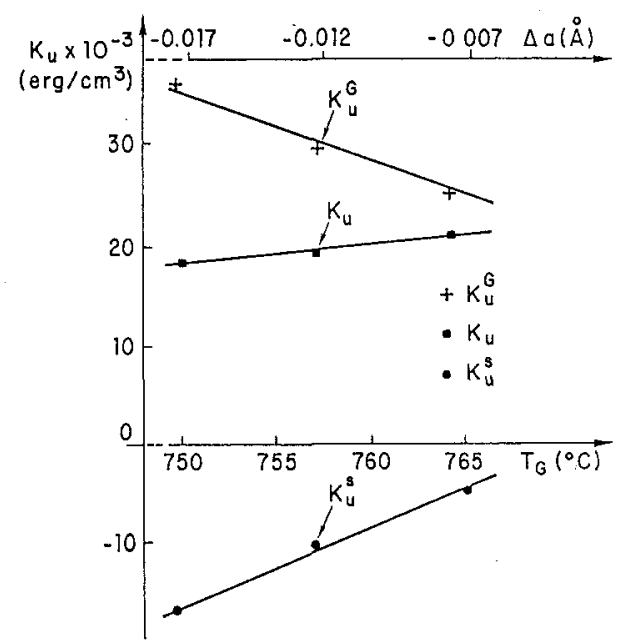

Fig. 4. Variation in $K_{u}$ as a function of growth temperature $\mathrm{T}_{\mathrm{G}}$. Melt $\mathrm{A}^{\mathrm{u}}$ 


\begin{tabular}{|c|c|c|c|c|c|c|}
\hline MELT & $\begin{array}{c}T_{G} \\
(\circ \mathrm{K})\end{array}$ & $\begin{array}{c}\mathrm{Ku} \\
\left(\mathrm{erg} / \mathrm{cm}^{3}\right)\end{array}$ & $\begin{array}{c}4 \pi \mathrm{M} \\
(\mathrm{Gauss})\end{array}$ & $\begin{array}{c}I \\
(\mu \mathrm{m})\end{array}$ & $Q$ & $\begin{array}{c}\mu_{\mathrm{W}} \times 10^{-3} \\
\left(\mathrm{~cm} . \mathrm{s} . \mathrm{Oe}^{-1}\right)\end{array}$ \\
\hline \multirow{3}{*}{$\mathrm{A}$} & 1038 & 21300 & 206 & 0,48 & 12,6 & 0,723 \\
& 1030 & 19600 & 240 & 0,36 & 8,6 & 0,695 \\
& 1023 & 18300 & 300 & 0,26 & 5,2 & 0,690 \\
\hline \multirow{2}{*}{$\mathrm{B}$} & 1023 & 6400 & 340 & 0,13 & 1,4 & 1,400 \\
\hline
\end{tabular}

Table 3 - Magnetic properties of GdIG : Bi Pr Al films.

from a higher praseodynium content in the melt (melt B). Compare to the previous compositions (Table 3) the domain wall mobility $\mu_{\omega}$ is lower due to the rare earth ions which increases the magnetic relaxation effects but the values of $\mu_{w}$ are high enough for devices applications.

2-4. The feasibility of the magnetooptical transducer was performed on a one channel mock-up [6]. The tape was recorded either conventionally or by Curie point writing of Chromium dioxide pigments in order to record very narrow tracks. The readout pick-up comprises :

- An HE-NE Laser

- A partially polarized beam splitter

- A.25 NA objective

- The magneto-optical transducer

- A polarizing beam splitter for rotation measurement

- Two optical detectors feeding a differentiel amplifier.

The partially polarizing beam splitter transmits about $90 \%$ of the light to the objective. It reflects only $10 \%$ of the returning beam towards the cells but $90 \%$ of the information amplitude (perpendicular to the source polarization) is reflected. The polarization detector compares the amplitude of both projections $45^{\circ}$ apart form the source polarization axis.

A second optical configuration has been tested : a conventional polarizing beam splitter and quarter wavelenght plate were used to reflect nearly $100 \%$ of the light toward the cells. Instead of detecting the polarization rotation (which in this configuration does not exist), two cells detect the far field shift due to the index difference between spin up and down domains. The highest recorded frequency to date corresponds to $16 \mathrm{kFCI}$ on a 3 microns track i.e. 20 Mbits per square centimeter. The measured $\mathrm{S} / \mathrm{N}$ ratio at $8 \mathrm{kFCI}$ is $16 \mathrm{~dB}$ (RMS/RMS) over $16 \mathrm{kFCI}$ Band width. This is slightly above the decoding threshold. Compared to a conventional head with a $1 \mathrm{~mm}$ traek width, the $\mathrm{S} / \mathrm{N}$ ratio is $7 \mathrm{~dB}$ lower. By introducing a $25 \mathrm{~dB}$ correction to account for the track width ratio, a $18 \mathrm{~dB}$ advantage to the optical head is obtained.

\section{CONCLUSTON}

The feasibility of an optical readout of magnetic has been shown using thin garnet film transducers. Two solutions have been investigated : either from heat treatment which reduces $K$ or by doping the films with ions which induce in-plane magnetization. Materials with optimum physical parameters and good surface quality as required in device application can be monitored from Bi Pr Al - substituted GdIG. Such a device enables the realization of a high data rate fixed head recorder. As an example : one Gigabit per second can be obtained by parallel reading of 2000 tracks on a half inch tape running at 20 ips. Of course such a recorder also needs a recording headstack. Work is going on integrating some 2000 record-only heads using thermally induced effects.

\section{ACKNOWLEDGMENTS}

We wish to thank M. ARTINIAN for magnetooptical and magnetic measurements and $M$. ROMMELUERE for electron mocroprobe analysis.

\section{REFERENCES}

[1] Tatsuo NOMURA, IEEE transactions on Magnetics Mag.21, N 5, (Sept. 85), 1545.

[2] T. YAMADA, H. MATSUDA, Y. MAKINO and M. HIMURO, Proceed of the Int. Conf. on Ferrites (Sept.80), 791.

[3] J. GOUZERH, H. LE GALL, Y. DE BEAUREGARD, D. GRATECAP and J.M. DESVIGNES, J. Appl. Phys., 55,(1984), 2566

[4] J.M. DESVIGNES, J.M. CERCEAU, V.B. KRAVTCHENKO and H. LE GALL, J. of Cryst. Growth, 14, (1979), 559

[5] B. FERRAND, M.F. ARMAND, H. MORICEAU, J. DAVAL, J.C. GAY, Mat. Res. Bull, 21, (1986), 633.

[6] J.P. CASTERA and J.C. LEHUREAU, Presented at Intermag Conf. Tokyo. April 87 